\title{
Über die Einheit des Menschen aus ärztlicher Sicht
}

Gahl, Klaus

Veröffentlicht in:

Jahrbuch 2001 der Braunschweigischen Wissenschaftlichen Gesellschaft, S.27-39

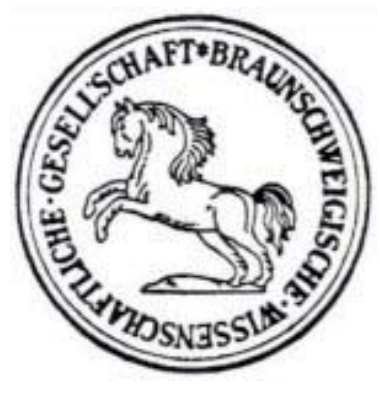

J. Cramer Verlag, Braunschweig 
KLaus GaHL, Braunschweig

\title{
Über die Einheit des Menschen aus ärztlicher Sicht
}

\author{
Braunschweig, 06.04.2001*
}

Der folgende Vortrag geht zurück auf einen Beitrag zu einem Workshop „Das HirntodKriterium und die Einheit der Person“ am Philosophie-Institut der Fern-Universität Hagen ${ }^{1}$, der am 8.9.95 vor dem Hintergrund der damals intensiv auch in gesundheitspolitischen Gremien geführten Diskussion der Frage „Wann ist der Mensch tot?“ abgehalten wurde. Das Kernthema des Hirntodes wurde seinerzeit von dem Münchener Neuropsychiater Prof. Dr. med.Angstwurm vorgetragen. Mir war die Aufgabe gestellt worden, aus ärztlicher Sicht über die im unmittelbaren Umgang mit kranken Menschen erfahrbare Einheit der Person vorzutragen. Ich habe seinerzeit versucht, das an den für den Arzt handlungsleitenden Begriffen Anamnese, Diagnostik und Therapie aufzuhängen. Heute sollen diese Schritte um den der begründbaren Vorausbeurteilung, der Prognose, erweitert werden.

Es handelt sich dabei nicht um allgemeine Lehrmeinung, nicht um eine empirisch belegte, gar statistisch gesicherte Erfahrung, vielmehr um einen anthropologischen Zugang, z.T. um vorwissenschaftliche Erfahrung, die aber sehr wohl systematisierbar und vermittelbar ist. Darin sehe ich die für Medizinstudenten und Ärzte potentiell pädagogische Zielsetzung dieser Reflexion.

Die „Einheit des Menschen“ soll hier nicht aus der theoretischen Distanz einer wissenschaftlichen Disziplin (,der Medizin“) dargestellt werden, vielmehr als konkret und unmittelbar erfahrbare phänomenale Einheit, derer wir im ärztlichen Umgang mit kranken Menschen alltäglich ansichtig werden können. Hier schon der erste Einhalt! Ihrer ansichtig werden zu können, impliziert doch die Möglichkeit, diese Einheit gerade nicht wahrzunehmen: sei es konzeptionell in eindimensionaler Sicht reduktionistisch naturwissenschaftlicher Medizin, sei es durch organisatorische Umstände bedingt (z. B. durch Personal-, d. h. Zeitbudgets) oder auch institutionell (mit fachspezifischer Funktionsverteilung) oder sei es auch, daß die Einheit des Menschen auf Seiten des Kranken absichtlich verborgen bleibt. Auf Begründungen und die Berechtigung dieser unterschiedlich motivierten oder bedingten Reduktionen oder auf den vielleicht nötigen beiderseitigen Selbstschutz absichtlicher oder unfreier Selbstverborgenheit des Kranken einzugehen, würde hier zu weit führen. Wünschenswert bleibt es, die Reduktion jeweils als solche zu sehen und den Stachel des Unvollständigen, des Desiderates zu spüren.

\footnotetext{
* Vortrag vor der Plenarversammlung der Braunschweigischen Wissenschaftlichen Gesellschaft

${ }^{1}$ Workshop „Das Hirntodkriterium und die Frage der Einheit der Person“ des PhilosophieInstitutes der Fern-Universität Hagen am 08.09.1995; Leitung Prof. Dr. J. P. Beckmann, später in Zschr Ethik in der Medizin (1999): 11: 2-11
} 
„Einheit des Menschen“ aus ärztlicher Sicht ist dargestellt worden in verschiedenen Modellen, die den cartesianischen Dualismus psycho-physisch parallelistisch oder psychosomatisch oder mit dem bio-psycho-sozialen Modell von Thure von Uexküll ${ }^{2}$ zu überbrücken versuchen. Derartige Modelle werden jedoch innerhalb der Medizin weithin wohl als für funktionelle oder sog. psychosomatische Erkrankungen relevant angesehen, nicht aber für die alltägliche Begegnung mit kranken Menschen jedweder medizinischen Fachrichtung. Der folgende Beitrag will dagegen Aspekte der Einheit des Menschen aufspüren in den genannten ärztlichen Handlungsschritten, wie sie - wenngleich in unterschiedlicher fachgebotener Spezifizierung - prinzipiell in allen klinischen Disziplinen ähnlich praktiziert werden. Meine Überlegungen gehen von der unmittelbaren Wahrnehmung des kranken Menschen in seiner Leibgestalt aus, verfolgen seine Kranken- und Krankheitsgeschichte als Stück einer Biographie und führen zur Sinnfrage der Therapie und den Entwurf auf Zukunft hin.

\section{A) Erfahrung der Einheit des Menschen in der Diagnostik:}

Die wohl häufigste Primärsituation einer Arzt-Patient-Beziehung ist die, daß ein Kranker, ein infolge eines Leidens medizinischer Hilfe bedürftiger Mensch sich an einen Arzt wendet. Gemeinsam wird das aktuelle Beschwerdebild eruiert, erstellt, konstruiert: körperliche Mißempfindungen wie Schmerzen, Bewegungsbehinderungen, Leistungsminderung, Schwindel, Gewichtsverlust, Störungen vegetativer Funktionen etc. werden erfragt bzw. geschildert. Es folgt die sogenannte körperliche Untersuchung, ergänzt um medizinisch-technische Diagnostik. So der übliche Einstieg in die Untersuchung.

Wie wird hier „Einheit des Menschen“ für den Arzt sichtbar?

Der Kranke leidet mehr oder weniger unter körperlichen Beschwerden, er erlebt und erleidet sie und sich selbst konkret, sei es als eine Verletzung, vielleicht als unbestimmtes Mißbefinden, als eine Organschädigung oder eine Störung des Gesamtorganismus. Erleben, Befinden, Leiden sind ja nicht allein körperliche Phänomene, auch wenn der Kranke sie überwiegend in der Sprache der Körperlichkeit formuliert. Sie sind ebensowenig rein seelische Phänomene. Das Sich-Befinden, das reflexive Verhältnis des Kranken, des Leidenden zu sich selbst, weist schließlich darauf hin, daß es hier nicht um rein objektivierbare Körperlichkeit geht. Vielmehr ist es unsere Leiblichkeit, die sich vordergründig ,rein somatisch“ zeigt, in der aber Empfindung, Selbstwahrnehmung, Ausdruck und Handlung zum Vorschein kommen.

Vier ,Stufen“ oder Dimensionen der Leiblichkeit - keineswegs gegeneinander abgrenzbar und auch nicht in hierarchischer Ordnung zueinander - sollen hervorgehoben werden:

1) die Stufe der Spontaneität, wie sie sich in Eigensteuerung, Haltung, ungerichteter Bewegung, in Gestik und Mimik zeigt. Schon auf dieser „Stufe“ tritt der Leib aus der

2 Thure von Uexküll \& Wolfgang Wesiack (1988): Theorie der Humanmedizin - Grundlagen ärztlichen Denkens und Handelns. München - Wien - Baltimore, Verlag Urban \& Schwarzenberg 
Isolation, aus der Vereinzelung heraus auf Beziehung hin. Mit der inneren, der nicht mentalen Selbstwahrnehmung (Propriozeptivität) und der Reagibilität biologischer Anpassung an innere (z.B. Gleichgewichtsauslenkungen, Belastungen des Bewegungsapparates etc.) oder äußere Signale wird ein Zusammenspiel zwischen Leib und Umwelt deutlich.

Auf dieser Stufe bereits drückt sich Krankheit nicht allein als Körperphänomen sondern auch Kranksein, Leiden als persönlich erlebte und gestaltete Veränderung aus, - wahrnehmbar für uns alle in unmittelbarer Anschauung, z. B. in der Leidensmiene oder -haltung; für den Arzt unter Umständen speziell interpretierbar auf eine bestimmte, definierbare Krankheit mit ihrer charakteristischen Leibgestalt hin: seien es so unterschiedliche Erkrankungen wie eine den Körper stark verändernde Polyarthritis oder eine Depression.

2) die Stufe der Reflexivität, des Sich-Erlebens, Sich-Empfindens, des Sich-Wohl- oder Mißbefindens. So wird der Mensch z. B. im Schmerz sich seiner selbst inne, nicht als rationale Selbstvergewisserung im „,cogito - ergo...“, sondern eher wie der Träumer, der Erwachende, der sich sozusagen der „Realität“, seines Wachseins vergewissert, indem er sich selbst kneift. Es ist die Erfahrung der „,selbst-referentiellen Eigenständigkeit“" (P. Christian $\left.{ }^{3}\right)$, die durch die Leiblichkeit ermöglicht wird und durch sie erlebt werden kann.

Auf dieser Stufe erleben wir Leibgefühle wie Hunger und Durst, Müdigkeit, Anspannung, Erschöpfung, Schwindel, die Ekstase leiblicher Hingabe. Diese Leibgefühle sind ja alle nicht ,rein somatisch“, objektivierbar, körperlich erklärbar, schon gar nicht darauf reduzierbar. Ebensowenig das Sich-Krank-Fühlen, das der leiblichen Selbstvermittlung bedarf. Sie sind allesamt, auch das Sich-krank-Fühlen individuell und situativ beeinflußte Leibgefühle, aktiv gestaltete Leibgefühle. Der Kranke ist darin (auch) Subjekt, Akteur seines Krankseins.

Wie das Mißempfinden leiblich spürbar wird, so kann sich andererseits Kranksein auch darin äußern, daß die Möglichkeit reflexiven Sich-Empfindens gerade verlorengeht bis hin zu passageren oder länger anhaltenden oder gar permanenten Entfremdungsgefühlen oder zur sensorischen und emotionalen, ja sogar kognitiven Beziehungsleere zum eigenen Körper oder der Depersonalisation ${ }^{4}$ in der Depression. Die dem Begriff der Depersonalisation implizite Verankerung der Personalität in der Fähigkeit, sich seiner selbst in der eigenen Leiblichkeit zu vergewissern, sei hier nur angedeutet. Sie scheint mir aber für das Thema der „Einheit des Menschen“ zentral. Auch nach Schock oder Narkose, ja nach kurzer Bewußtlosigkeit z.B. durch eine Gehirnerschütterung kann das Gefühl für den eigenen Leib vorübergehend gestört sein. Derartige Störun-

3 Paul Christian (1989): Anthropologische Medizin. Berlin - Göttingen - Heidelberg - New York, Springer-Verlag, p 295

4 Viktor Emil von Gebsattel (1954): Zur Frage der Depersonalisation. In: ders.: Prolegomena einer medizinischen Anthropologie. Berlin - Göttingen - Heidelberg New York, SpringerVerlag, pp $18-46$ 
gen können partiell und hinsichtlich unterschiedlicher Funktionen (z.B. Sensibilität und Motorik) dissoziiert auftreten und über unterschiedlich lange Zeit anhalten.

- Zitat eines 93jährigen Mannes: er habe sich nach einer Abdomen-Computertomografie ,erholen“ müssen, da in der und durch die Untersuchung „Körper und Geist getrennt worden" seien.

- Meisterhaft und spannend geschildert von Oliver Sacks ${ }^{7}$ in seinem Bericht über die Folgen eines eigenen Bergunfalls: „Der Tag, an dem mein Bein fortging.“

In milderer Form läßt sich auch bei gesunden Menschen häufig eine merkwürdige Dissoziation oder Abtrennung leiblicher Selbstempfindungsfähigkeit beobachten.

3) Leiblichkeit äußert sich auch als Intentionalität, auf Außenwelt, Umwelt, auf Mitwelt gerichtet. Intentionalität ist hier nicht verstanden als rationale Absicht, vielmehr als präreflexive Intentionalität leiblicher Ausrichtung: in der sinnesphysiologisch organisierten Wahrnehmungsfähigkeit (hier korrespondiert der Intentionalität die Rezeptivität: dem Auge das Licht, dem Ohr der Klang, dem Tastsinn die Berührung), in der gezielten, aber nicht nur in der bewußten Bewegung, in der antwortenden Gestik und Mimik. Die Sexualität zeigt diese intentionale Leiblichkeit besonders deutlich. Der Psychiater Viktor Emil von Gebsattel ${ }^{5}$ spricht in seinen „Prolegomena einer medizinischen Anthropologie“ (1954) vom Geschlechtsleib. Es ist zu betonen, daß es sich dabei um eine unter anderen möglichen Aktualisierungsformen relationaler und intentionaler Leiblichkeit auf ein Gegenüber hin handelt. Der Leib ist - mit einem Begriff von E. Husserl - „fungierende Intentionalität“.

4) Auf der nächsten Stufe verschmelzen Intentionalität und Reflexivität zur Beziehungsfähigkeit, zur Empathie, zur Relationalität und Intersubjektivität, indem Leiblichkeit ermöglicht, daß der Mensch sich (reflexiv) als ... gerichtet auf... fühlen kann: als Mann, als Frau, als Mitmensch, als Tochter oder Sohn, als Vater oder Mutter, als Sportler, als Arbeitspartner (ich denke an das Bild der beiden Holzsäger, die systolischdiastolisch, muskelanspannend und entspannend, konsensuell - wie P. Christian ${ }^{6}$ es nennt - sich aufeinander einstellen), als Mitmensch. Es ist dies die soziale Dimension der Leiblichkeit.

Einen Begriff des Arztes und wichtigsten Förderers anthropologischer Medizin, Viktor von Weizsäckers ${ }^{7}$ aufnehmend und erweiternd möchte ich als weitere Stufe die der Transjektivität nennen, das „Sich-über-sich-hinaus-Werfen“. Weizsäcker sah im Verstehen des Gegenübers nicht zuerst einen rationalen, sondern einen ursprünglich personalen Akt: “...weil mein Verstehen gleichsam in den andern hinüberschlüpft, so wollen wir ... dieses Jemand-Verstehen ein transjektives nennen“8. Im Zusammen-

${ }^{5}$ Viktor Emil von Gebsattel (1954 ): Geschlechtsleib und Geschlechtstrieb. In: ders.: Prolegomena ... a.a.O. pp 314 - 329

6 Paul Christian (1989): Anthropologische Medizin, a.a.O.

7 Viktor von Weizsäcker (1926): Der Arzt und der Kranke. In: Gesammelte Schriften, Bd. 5. Frankfurt a.M. 1987, Suhrkamp-Verlag, pp 9-26

8 a. a. O., p 20 
hang mit der leibgebundenen Intersubjektivität soll mit dem Begriff der Transjektivität jedoch nicht das rationale Verstehen, vielmehr das ,Sich-Erleben auf den Anderen hin“ beschrieben werden, nicht ausschließlich auf der mentalen, gar nur der rationalen Ebene, sondern in der unmittelbaren empathischen Zuwendung, die uns manchmal geradezu wie eine Erschütterung (im Glücksgefühl) durchströmt. Es ist dies die Stufe des Sozial- oder Rollenleibes, in dem und durch den wir uns als bezogen auf...., als relational erleben. In schönster Weise in der erotisch-sexuellen Verschmelzung.

Hier sei auch - der Sexualität sehr nahe - die Scham genannt. Abwehr- und Selbstbewahrungsinstinkt, offenbart und verbirgt sich in ihr zugleich das reflexive Selbstempfinden: zugleich Subjekt und Objekt, auch in seiner Verletzbarkeit. Wird sie nicht wahrgenommen und in ihrer schützenden Funktion nicht geachtet, wie das m.E. auch geschieht in objektivierender Reduktion des Kranken auf seine Körperlichkeit, so wird die Einheit des Menschen gefährdet. Auf die "Spannung" sach- und personbezogen notwendiger, schützender Beschränkung, Reduktion, sei nachdrücklich hingewiesen. Körperlichkeit ist nur in der Objektivierung von außen als „unbeseelt“ zu sehen. Von innen her, in unserem Befinden, unserem Selbsterleben, subjektiv sind und erleben wir uns leiblich. Auch das sog. rein Psychische bedarf der leiblichen Vermittlung: „Nichts Seelisches hat keinen Leib.“(Viktor von Weizsäcker). Der bei Kranken und Sterbenden oft zu beobachtende Prozeß des krankheitsbedingten und krankhaften Schamverlustes, in dem auch die Persönlichkeit des Kranken unterzugehen scheint, darf nicht dazu verführen oder gar vermeintlich dazu berechtigen, den Kranken als Objekt, als „Körperding“ zu behandeln.

Auch diese Stufe intentionaler und relationaler Leiblichkeit ist kränkbar: im Verlust der sich selbst erlebenden Beziehungsfähigkeit, mag er mehr somatisch (z. B. die sogenannnte organisch bedingte Impotenz) oder psychisch manifest werden (in der Depression). Selbst in alltäglichen Schilderungen von Kranken kann er sich äußern: z.B. in der Identitätskrise vieler Herzinfarktpatienten, im Verlust des Selbstwertgefühles (so eine Mittvierzigerin ein Jahr nach Brustamputation). Es genügt hier für ein adäquates Krankheitsverständnis meines Erachtens nicht, der naturwissenschaftlichen Kategorie von Körperlichkeit die des seelischen Erlebens, der psychischen Verarbeitung zu addieren. Es ist das eine Kranksein, die Einheit des Menschen, die auch in ihrer Leiblichkeit verankert, konstituiert ist, derer wir als Ärzte ansichtig werden können im alltäglichen Schritt der körperlichen Untersuchung, der Diagnostik. Es geht nicht um die Verortung der Einheit des Menschen, des Subjektes oder der Person in speziellen, kognitionsbezogenen Hirnstrukturen und nicht um Bewußtseinsakte, somit auch nicht um eine kognitivistische Reduktion der Einheit des Menschen auf mentale Hirnleistungen (z.B. aktuelle Selbstbestimmungsfähigkeit als aktualisierte Autonomie); auch nicht um ein hirnorganisch zu verortendes integratives, selbstregulatorisches Funktionsmodell. Es geht vielmehr um die Selbsterfahrung, das Selbsterlebnis als das einer Einheit im Leiblichen.

Wenn auch die skizzierten Dimensionen der Leiblichkeit in individuell unterschiedlicher und wechselnder Intensität erlebt werden, so sind sie doch nicht alle bewußtseins- 
abhängig. Auch die Amöbe läßt - ihrer biologisch einfacheren Organisation entsprechend in weniger entfalteter Ausprägung Aspekte von Leiblichkeit erkennen. Der menschliche Organismus ermöglicht eine intensivere, reichere Entfaltung u. a. aufgrund der hirnorganischen Speicherung von Sinneseindrücken i.w.S., von subkortikalen und kortikalen Verknüpfungen, der Assoziations- und Integrationsfähigkeit und des Erinnerungsvermögens, die dem Menschen eine im Tierreich sonst nicht erreichte Bewußtseins- und damit auch Selbsterlebnispotenz eröffnet ${ }^{9}$. Bewußtsein, die sog. geistige Dimension des Menschen, ist dabei ebenso an Hirnstrukturen und -materie gebunden wie die emotionale oder perzeptive Fähigkeit, ,Kontakt“ aufzunehmen mit der Mitwelt. Eine Trennung ist nicht möglich, wohl aber eine Unterscheidung geistiger und materieller Phänomene der Leiblichkeit. Wie wollte man auch in Sprache oder geplanter Handlung die leibliche und geistige Dimension trennen?! Beide - Sprache und Handlung - sind auch gegenwartsübergreifend gespannt zwischen (psychologisch) „Motiv“ und „Zielerreichung“, (anthropologisch) Entwurf und Vollendung, (philosophisch) Retention und Prolepsis.

Die „nicht-objektivierbare Körperlichkeit“ oder die nicht auf Körperlichkeit reduzierbare Leiblichkeit weist in eine zweite Richtung, in der der Arzt in der Begegnung mit dem Kranken der Einheit des Menschen ansichtig werden kann. Der Kranke ist in seinem leiblichen Kranksein (und daran ist hier vor allem gedacht) Subjekt, d. h. Akteur, spontan und reaktiv Handelnder, Agierender seines Krankseins, wie er auch der Krankheit unterworfen (sub-iectus) ist. In wechselnder Gewichtung ist er aktiv und passiv zugleich, hat und gestaltet er seine (!) Krankheit, besser: sein Kranksein, darin dialektisch frei und leidend. In der Schmerzempfindung, auch in anderen Leibgefühlen wie Hunger und Durst, Erschöpfung und Frische u. a. wird uns das besonders deutlich: sei es willentlich oder emotional oder affektiv gesteuert, erleben oder erleiden und gestalten wir in kranken und gesunden Tagen das Mißbefinden stärker oder schwächer. Nicht nur die stark vom sog. vegetativen Nervensystem abhängigen Leibgefühle, nein, wir können sogar den Ablauf von „Organkrankheiten“, selbst die Heilung von Knochenbrüchen mental beeinflussen.

Hier verrät sich eine Wechselbeziehung, eine Interdependenz von Handeln und Leiden: zu leiden ist nicht nur passiv, sondern auch aktiv; ein Leiden anzunehmen, es zu bewältigen, vielleicht als Wandlung, als biographische Notwendigkeit, als Schwelle zu erleben (Buytendijk ${ }^{10}$ und nach ihm Christian ${ }^{11}$ sprechen von der Schwellenpositionalität der Krankheit) - das alles sind ja aktive Umgangsmöglichkeiten mit dem Leiden. Umgekehrt ist Handeln nicht nur aktiv, es kann - und das verrät sich häufig in Krankheitszuständen auch eine Leidensform sein.

9 s. Gerhard Roth (1994): Das Gehirn und seine Wirklichkeit. Frankfurt a. M., SuhrkampVerlag

10 Frederik J.J.Buytendijk (1967): Prolegomena einer anthropologischen Physiologie. Salzburg, Otto Müller Verlag, p 160

11 Paul Christian (1989), a.a.O. 


\section{B) Erfahrung der Einheit des Menschen in der Anamnese:}

Ist mit der unmittelbaren Krankenuntersuchung, diesem einleitenden diagnostischen Schritt, die vergleichsweise statische Leibgestalt der Krankheit und des Krankseins deutlich geworden, so wird mit dem Handlungsschritt ${ }^{12}$ der Anamnese die Entwicklung, die dynamische Zeitgestalt der Krankheit, diese eher im Sinne eines idealtypischen Konstruktes, erarbeitet - durch den Arzt transitiv, vom Kranken reflexiverinnernd. Die von uns Ärzten meist sogenannte Krankengeschichte ist meist ein reduzierendes Datieren von Vorkrankheiten, Operationen oder anderen medizinisch relevanten Ereignissen, allenfalls noch die Entwicklung einer Krankheit, aber höchst selten die Geschichte eines Kranken oder eines Menschen in seiner Erkrankung und seinem Kranksein.

Indem ihre kausale oder konditionale Beziehung zu Entwicklungsphasen, Perioden oder anderen biologischen Rhythmen berücksichtigt wird, kann der situationsabhängige oder lebensgeschichtliche Zusammenhang einer Krankheit, eines Krankseins erkennbar werden; mehr noch, wenn Aktualkonflikte oder belastende, kränkende (sic!) Schlüsselereignisse oder Lebenssituationen, wenn neben der körperlichen auch die seelische und geistige Entwicklung, die Sozialisation in Familie, Ausbildung und Beruf und Gemeinschaften beachtet werden, soweit diese Bereiche das Kranksein, auch die „Verarbeitung“ von Krankheit, prägen oder geprägt haben.

Die Zeitgestalt des Krankseins gewinnt in der biographischen Anamnese eine andere Qualität als die des physikalischen und biologischen Zeitablaufes der Krankheitsgeschichte. Die Zeit des Krankseins ist ,ein Stück Lebensgeschichte“ (Siebeck 1949 ${ }^{13}$ ), ist biographische Zeit mit der Offenheit für Selbsterfahrung, für eine gesteigerte Empfänglichkeit und Verletzbarkeit, für die Erfahrung der eigenen Zeitlichkeit und Geschichtlichkeit, auch hin auf den eigenen Tod. Es ist wieder die mögliche „Schwellenpositionalität“ der Krankheit, besser: des Krankseins, in der Biographie des Kranken.

Zeit hat hier neben und in der extensiven und intensiven Zeiterfahrung auch eine existentielle Erlebnisqualität von Endlichkeit und Vergänglichkeit, d.h. auch mehr oder weniger ständigem Bedrohtsein ,,mitten im Leben“. Die Geschichte, die Zeitgestalt der Erkrankung, des Leidens, wird schließlich mitgeprägt von der Selbstinterpretation (,health belief“): Was denkt, was empfindet der Kranke selbst hinsichtlich der „Bedeutung“, des Sinnes seines Krankseins? Sie wird auch mitbestimmt von der Einrichtung mit dem Kranksein, das auch Gewinn bringen kann, Entlastung von Aufgaben und Verpflichtungen, den Gewinn der Regression. So hat die individuelle Erkrankung immer auch eine soziale Dimension.

Mit dem Erleben, dem Erleiden des Krankwerdens ist oft ein verändertes Zeiterleben verbunden: intensiver oder konturlos, das Erlebnis der Langeweile oder Zeitleere oder Zeitraffung, mit der Vergegenwärtigung des Vergangenen oder mit dem Versuch, die Zukunft einzuholen, oder sich (wieder) in die Zeit einzuorten.

12 Indem die Anamnese als Handlung verstanden wird, öffnet sie sich als solche auch der ethischen Reflexion, ihrer Begründung und Rechtfertigung.

13 Richard Siebeck (1949): Medizin in Bewegung. Stuttgart, Georg Thieme Verlag 
Was in der dreischichtigen Anamnese der Krankheit, des Kranken und seiner Erkrankung zum Vorschein kommt, ist ein mehrdimensionales Verständnis auch sogenannter rein somatischer Erkrankungen: auch sie haben einen biographischen und psychosozialen Stellenwert.

Hier die kurze „Anamnese“ eines 45jährigen promovierten Akademikers, der uns nach einem akuten Herzinfarkt zur Herzkatheteruntersuchung überwiesen wurde. Die Diagnose des Infarktes wurde bestätigt; die Angiografie zeigte eine schwere diffuse Drei-GefäßKoronarkrankheit, die zur raschen Operation (4fach-Bypass) veranlaßte. Der Verlauf war weitgehend komplikationslos, die postoperative Mobilisierung bei uns und in der Anschlußheilbehandlung trotzdem verzögert. - Zur Vorgeschichte: ohne die Standard-Risikofaktoren (erhöhter Blutdruck, Rauchen, Fettstoffwechselstörungen) hatte der strebsame, leistungsbewußte und zum Perfektionismus neigende Psychologe „gesundheitsbewußt“ gelebt, gejoggt. Der Infarkt, ohne angegebene Vorboten, war ihm wie ein „Unfall“ vorgekommen. „Wie konnte das passieren? - mir, der ich so gesundheitsbewußt gelebt habe?" Wie es denn jetzt weitergehen könne mit der Arbeit, dem Beruf, dem Sport? Äußerungen mit Blick auf die Ehefrau, auf Berufskollegen. Herr Dr. X stand unter dem Druck, innerhalb einer befristeten Anstellung am Universitätsinstitut seine Habilitation zu erreichen, wenn er nicht in eine ihm niedriger erscheinende Tätigkeit als Schul- oder Betriebspsychologe absteigen sollte. Jetzt aber durch den Infarkt und die Operation mindestens 4 Monate aus der Arbeit herausgerissen! Und wie weiter?

Diese Krankengeschichte macht auch ohne psychosomatische oder psychoanalytische Interpretation deutlich, wie stark selbst (im naturwissenschaftlichen Verständnis) ,rein somatische"Krankheiten wie der Herzinfarkt eine psychosoziale Dimension, auch die der biographischen Einheit des Menschen, haben. Sie ist es, die in dem ärztlichen Handlungsschritt der Anamnesenerhebung zum Vorschein kommen kann. In der transitiv und reflexiv geleiteten und geleisteten Erinnerung erlebt der Kranke seine biographische Identität, in „zeitübergreifender Vergegenwärtigung“ (Auersperg). Hier ist zunächst an die Rückholung, die erinnernde Vergegenwärtigung gedacht. Es kann darin auch die Macht, die kränkende Wirkung des vermeintlich Vergangenen deutlich werden, besonders, aber nicht ausschließlich in der psychoanalytischen Aufarbeitung von Verdrängungen in der Biographie.

\section{C) Erfahrung der Einheit des Menschen in der Therapie:}

Hier komme ich zu dem aus der Sicht des Kranken zentralen Handlungsschritt: der Therapie. Sie ist ihm das wichtigste Anliegen, wenn er sich an einen Arzt wendet. Was aber ist ihm, dem Kranken, das Ziel, der Sinn der Therapie? Und was ist es für den Arzt?

Therapie zielt auf Heilung, auf Leidensminderung, auf physische Restitution und soziale Reintegration, auf Wiederherstellung von Gesundheit - idealiter in ihrer integralen Form „körperlichen, seelischen und sozialen Wohlbefindens“ (gemäß der Definition von Gesundheit nach der Welt-Gesundheits-Organisation WHO). In der leiblich konstituierten Intentionalität und Relationalität, im Rollenleib, wurde die soziale Dimension bereits angesprochen. „Soziale Reintegration“ umfaßt auch diesen Aspekt, nicht nur die Wiedereingliederung in Familie oder Arbeitswelt.

Obwohl mit der WHO-Definition ein m. E. nicht realisierbarer Gesundheitsbegriff statistisch wie auch moralisch und pragmatisch normativ gesetzt wird für das Handeln, das Behandlungsziel des Arztes, so ist doch zu beachten, daß diese Definition die drei Di- 
mensionen bio-psycho-sozialer Einheit des Menschen (wie sie dem Uexküll'schen Krankheitsverständnis zugrunde liegt) umfaßt und sie als reflexive Befindlichkeit, als Wohlbefinden formuliert, das wohl schwerlich ,,von außen“ zu beurteilen ist. Und doch ist auch die objektive Intaktheit normaler Funktion und Struktur, Morphologie und Physiologie mitgedacht. Diese Intaktheit ist wiederum an der im sozio-kulturellen Umfeld als gesund eingeschätzten Normalität ausgerichtet. Das Wohlbefinden allein reicht nicht aus für eine Definition, nicht einmal für eine Beschreibung von Gesundheit.

In den letzten ca. 15 Jahren hat der Begriff der Lebensqualität die normative Funktion des Begriffes des Wohlbefindens abgelöst, indem skalierbare, meßbare, statistisch verrechenbare Daten mit ihrer scheinbaren Objektivität erfaßt werden. Mit der Lebensqualität, auf deren konzeptionelle, empirisch-methodische, statistische und interpretative Problematik ${ }^{14}$ hier nicht eingegangen werden kann, werden neben der krankheitsbedingten körperlichen Beeinträchtigung auch die seelische Belastung, das Leiden, die soziale Selbständigkeit und Abhängigkeit beurteilt. Diese Facetten des Krankseins zu verbessern durch kurative oder palliative Maßnahmen, ist das Ziel der Therapie akuter oder chronischer Krankheiten.

So sehr eine Quantifizierung von Minderung oder Verbesserung von Lebensqualität auch gutzuheißen ist, so kann sie doch auch den Blick auf die nicht meß- oder skalierbare Leidenserfahrung (sei sie positiv oder negativ), vielleicht auch Sinnerfahrung in der Erkrankung verstellen. Auch ist Lebensqualitätsminderung, eine Einschränkung körperlichen, seelischen und sozialen Wohlbefindens im Hinblick auf ein Therapieziel passager oder permanent erduldbar und im Einvernehmen (,,informed consent") auch zumutbar, ja geradezu nötig, wenn z. B. die schwere Beeinträchtigung, ja vitale Gefährdung durch eine zytostatische Therapie, oder auch eine riskante Operation in Kauf zu nehmen ist in der Hoffnung auf eine Überwindung oder Besserung eines Leidens. Damit erhebt sich die Frage der Vorrangigkeit von Behandlungszielen: geht es um momentane Verbesserung der individuellen und sozialen Befindlichkeit oder um erwartbare Lebensverlängerung mit vielleicht reduzierter Lebensqualität?

Therapieziel und Lebensziel, Heilungsabsicht und -erwartung des Kranken und des Arztes werden nicht immer kongruent, auch für den Kranken selbst nicht konstant, konsistent in ihrer inhaltlichen Charakterisierung oder ihrer rationalen oder affektiven Begründung sein. Lebensalter, die individuelle und soziale Situation des Kranken, die Krankheit mit ihrer spezifischen Prognose, die Verhältnismäßigkeit von einzusetzenden Behandlungsmaßnahmen und möglichem Erfolg werden Handeln und Duldung mit bestimmen.

An dieser Stelle sei eine kurze Krankengeschichte eingefügt, aus der die Inkongruenz von Therapieziel und -erwartung, aus der Sicht des Kranken geradezu die Inakzeptabilität von Behandlungsmöglichkeiten spricht:

14 Hans-Heinrich Raspe (1990): Zur Theorie und Messung der 'Lebensqualität' in der Medizin. In: P.Schölmerich \& G.Thews (Hrsg.): 'Lebensqualität' als Bewertungskriterium in der Medizin. Stuttgart - New York, G. Fischer Verlag, pp 23-40 
Ein 69jähriger dynamischer, körperlich bis vor kurzem gut leistungsfähiger Mann wird wegen instabiler Angina pectoris auf die Intensivstation (1985) eingewiesen. Gleich im ersten Gespräch äußert er den dringenden Wunsch nach einer Herzoperation; mit seinen Schmerzen könne er so nicht weiterleben. Die Herzkatheteruntersuchung zeigt die linke Herzkammer stark erweitert mit einer weit ausgedehnten Infarktnarbe (der Patient wußte von keinem entsprechenden Ereignis) und einer generalisierten hochgradigen Kontraktionseinschränkung; zwei der drei Herzkranzgefäße verschlossen, das dritte hochgradig eingeengt. Inoperabel! Der Patient konnte unseren Beschluß, von einer Operation abzusehen und medikamentös zu versuchen, ihn weitgehend beschwerdefrei zu machen, nicht einsehen. Es müsse doch etwas zu machen sein, er könne jedenfalls so nicht weiterleben. Zwei Tage später verstarb er. - Ich frage, ob hier nicht mit der unabänderlichen Konsequenz des Todes leiblich eine existentielle Entscheidung getroffen worden ist in der Spannung zwischen Erwartung und Möglichkeit ärztlicher oder medizinischer Therapie? Auch eine Antwort auf die Sinnfrage eines eingeschränkten Lebens?

Läßt sich therapeutisch Gesundheit als ,völliges körperliches, seelisches und soziales Wohlbefinden" bei weitem nicht immer erreichen, so kann doch ärztliches Handeln auch auf die Akzeptanz von und auf den Umgang mit Einschränkungen, Behinderungen zielen - in unserer leistungsbezogenen Gesellschaft oft inkompatibel mit der Definitionsmacht und dem Handlungsdruck von patienteneigenen, von ärztlichen und von gesellschaftlichen Therapiezielen und -erwartungen, ja -forderungen. Gelingt dies, dann ist vielleicht auch durch die Überwindung von „Brüchen“ im Lebensentwurf, von Verlusten ein neu orientiertes Selbstwertgefühl wiederzuerlangen. Die Lebensqualität kann neue Facetten, neue Dimensionen gewinnen. Wie Kranksein ein Stück Lebensgeschichte, biographische Einheit, so kann auch mehr noch Gesundung, Heilung einen Schritt (mindestens potentiell) zur Sinneinheit des Menschen ermöglichen.

\section{D) Einheit des Menschen in der Prognose.}

Mit der Sinnfrage greift Therapie über die Gegenwart, über das akut notwendende Handeln im Blick auf Leidenslinderung oder -abwehr von Not und Bedrohung hinaus auf Zukunft, auf qualitative und quantitative Lebenserwartung. Von dieser mehr oder weniger weit vorausbeurteilenden Sicht, der Prognose her begründet und ggf. rechtfertigt der Arzt die Therapie. Er tut dies im ausdrücklichen oder notfalls im mutmaßlichen Einverständnis soweit möglich, nach Aufklärung des Kranken oder seines rechtmäßigen Vertreters, der in die Behandlung einwilligt. In diese einverständliche Zustimmung (im medizinischen Sprachgebrauch: den informed consent) geht etwas ein von dem Lebensentwurf des Kranken, der ja aus seiner Biographie erwächst, in den neben Hoffnung und Zuversicht, Lebenswille und Mut auch Befürchtungen und Ängste einfließen. Doch: Wie viele oder wie wenige Menschen leben heute gemäß einem aktiv gestalteten Lebensentwurf? Die meisten haben Erwartungen, auch Lebenserwartungen, darin auch eigene Prognosen, die sie vom Arzt bestätigt oder widerlegt wissen wollen. Dieses Wissen-Wollen ist sehr häufig zwiespältig: Nicht nur mit Blick auf das zu Wissende sondern auch wechselnd und relativ zur jeweiligen Situation in der Beurteilung des Lebenswertes.

Lebenserwartung ist aber auch Inhalt der medizinisch begründbaren, ärztlich zu vermittelnden Prognose, auch sie mit dem quantitativen und dem qualitativen Aspekt. Die quantitativ prognostizierte Lebenserwartung faßt die statistische Erfahrung krankheitsbezogener 
Lebensdauer nach Feststellung einer Krankheit (z. B. kleinzelliges Bronchialkarzinom 1,8 Jahre) und der Beurteilung des aktuellen Krankheitszustandes zusammen. Die Voraussage der Lebensqualität berücksichtigt viel stärker die individuelle Fähigkeit, mit dem Kranksein umzugehen, sieht Einschränkungen, Behinderungen und Schmerzen und Gefahren im weiteren Verlauf der Krankheit mit möglichen Aufs und Abs.

Es kann dabei auch zur Aufgabe des Arztes gehören, dem Kranken bei der von ihm zu leistenden Krankheitsbewältigung zu helfen, mit der es darum geht, das aktuelle Kranksein oder die überwundene oder die hoffentlich überwindbare Krankheit oder die erwartbaren Einschränkungen qualitativer wie quantitativer Lebenserwartung in die Zukunftsperspektive zu integrieren. Ja, er kann und muß diese Integration oft fördern. Die besonders in der zunehmend älteren Bevölkerung immer häufigeren nicht heilbaren chronischen Krankheiten erfordern das in steigendem Maße. Mit dieser Integration des Krankseins und der Krankheit (sic!: In der Spannung des pathischen Krankseins und der objektivierbaren Krankheit!) - mit der Integration in das weitere Leben „gehört Krankheit zur Individualisierung“. Ich zitiere Novalis ${ }^{15}$ : „Das Ideal einer vollkommenen Gesundheit ist bloß wissenschaftlich interessant. Krankheit gehört zur Individualisierung.“ Auch darin kann aus ärztlicher Sicht die Einheit des kranken Menschen deutlich werden.

\section{Zusammenfassung und Ausblick}

Betrachten wir die in den skizzierten Handlungsschritten von „körperlicher Untersuchung “, Erhebung der Anamnese, Therapie und Prognose vorscheinenden Aspekte der Einheit des Menschen unter dem Gesichtspunkt ihrer ethischen Relevanz, so lassen sich folgende Punkte herausstellen:

1. Die Leiblichkeit des gesunden wie des kranken Menschen ist „Organ“von potentiell auch personaler Selbstempfindung, auch möglicherweise selbstempfundener Einheit und Identität. Sofern der Leib als auf seine Gegenständlichkeit (Objektivierbarkeit) reduzierter Körper untersucht wird, bedarf es der sach- und wertorientierten Begründung (der Indikation). Der Kranke ist ja auch ein sich in seiner Leiblichkeit erlebendes und erleidendes wie auch gestaltendes Subjekt. Der dieser Dimension angemessene Umgang mit dem Kranken verlangt Achtung, Behutsamkeit und Respekt vor der Person des Kranken.

2. Anamnese ist Handlung und Ergebnis (der anamnestischen Exploration). Sie umfaßt idealiter die Geschichte einer Krankheit und die eines Kranken in seiner Erkrankung, in seinen Phasen des Krankseins. Geschichtlichkeit und Vergänglichkeit, Temporalität und Historizität, als Perspektiven der Zeitlichkeit können (mindestens fragmentarisch) in der erhobenen Anamnese als Einheit biographischer Kontinuität und Identität deutlich werden, vielleicht selbst in ihren Brüchen. Obwohl funktional geboten, auch zum beiderseitigen Schutz unabdingbar, ist doch der wechselseitige Reduktionszirkel

15 Novalis (1957): Werke in 4 Bänden, Bd. 1: Fragmente I: Die Enzyklopädie, Abteilung 5, p 284 (Heidelberg, Verlag Lambert Schneider), Fragment 1035 
skotomisierter Wahrnehmung (nur der Krankheit) und Ausblendung der biographischen Perspektive (Stichwort Schwellenpositionalität von Erkrankung!) problematisch und nicht wertfrei. Die Reduktion auf das funktional Gebotene und das dem Kranken und der Situation Zuträgliche und auf das der Kompetenz des Arztes Angemessene ist in der Achtung der Unverletzlichkeit der Person und der potentiell ansichtigen biographischen Einheit des Kranken aufgehoben.

3. Einheit des Menschen kann auch im Blick auf die Sinnfrage des Zieles ärztlicher Therapie deutlich werden, sofern diese über die bloße zweckorientierte Reparatur von Defekten hinausgeht und leibliche, seelische, geistige und soziale Leidensminderung, gar Heilung zu ermöglichen sucht. Hier ist die ethische Dimension wohl am deutlichsten zu erkennen. Geht es doch um die Wiedererlangung und Erhaltung von Gesundheit, um eine mindestens akzeptable Lebensqualität im individuellen wie im sozialen Bereich als hohen Werten. Soweit realisierbar, ist hier die Zielsetzung des Kranken zu beachten, durchaus mit der Möglichkeit divergierender Wertbegründungen des Kranken und des Arztes.

4. Die prognostische Beurteilung hat die quantitative und vielleicht mehr noch die qualitative Lebenserwartung, die Integration des Krank- oder von-Krankheit-Bedroht-Seins in der antizipierten Lebensspanne zu berücksichtigen. Das bedeutet nicht resignativen Pessimismus, sondern vernünftigen Realismus.

Der in dem gestellten Thema zu überbrückende „Körper-Seele-Dualismus“ scheint mir in der phänomenalen, dem Erlebnis zugänglichen Einheit des Leiblichen aufgehoben; jeweils als komplementär ergänzungsbedürftig können funktional die beiden „Partner“ getrennt wahrgenommen werden wie Welle und Korpuskel im Licht.

Mehr oder weniger verborgen und einer eindimensional naturwissenschaftlichen Sicht auf Krankheit verschlossen, können sich jedoch die herausgearbeiteten anthropologischen Konstitutiva (Leiblichkeit, Subjektivität, Zeitlichkeit, Sinnorientierung und Lebensentwurf) der unmittelbaren Begegnung, der vorwissenschaftlichen Erfahrung öffnen und auch im Blick auf eine medizinische Ethik handlungsrelevant werden - auch mit der unterschiedlichen Gewichtung ihrer jeweiligen Aspekte für den kranken Menschen und für den mit ihm betrauten und mit ihm umgehenden Arzt.

\section{Summary}

Discussions of , holistic “ medical practice often confine themselves to the treatment of socalled „psychosomatic disorders". This paper traces ways in which the patient's personal unity may become apparent to medical practitioners during three critical steps of everyday practice: physical examination, ,medical history“( (anamnesis) and treatment. Physical examination touches on the patient's „Leiblichkeit“ (his ,organ“ of bodily self-awareness of being a person). Objectifying of the „Leiblichkeit" may constitute an infringement upon the patient's personal unity and is only justified by strict medical indication. The patient not only is ill but he ,acts “ his illness (subject of and to his disease).-Medical history concentrates on the development of a disease but also has to keep in mind the potential 
biographical meaning of being ill and the patient's biographical identity. - The aim of medical treatment, finally, may touch on the patient's existential goals.-These three steps of medical practice are not discipline-specific, but span all areas of medical practice. It is argued that medical decisions should be guided by considerations of the patient's personal unity at each of the three steps. Prognostic judgement has to take into account quantitative and, more importantly, qualitative life expectancy, as well as the patient's ability to integrate an actual or potential illness into his or her life. In our view, taking all of these factors into account is not a sign of pessimistic resignation, but of realistic judgement.

Key words: self-awareness - physical examination - medical history - aims of treatment.

\section{Zusammenfassung}

Dem ärztlichen Handeln kann sich die phänomenale Einheit des Menschen in den drei Schritten der körperlichen Untersuchung, der Anamnesenerhebung und der Therapie erschließen. Die Leiblichkeit ist dem Menschen Organ (potentiell auch personaler) Selbstempfindung, auch möglicher selbstempfundener Einheit und Identität. Sofern der Leib als auf seine Gegenständlichkeit (Objektivierbarkeit) reduzierter Körper untersucht (diagnostiziert) wird, bedarf es der sach- und wertorientierten Begründung (der Indikation). Der Kranke ist als Subjekt, als Akteur seiner Leiblichkeit wie seiner Krankheit zu achten. - Die Anamnesenerhebung fragt idealiter nach der Entwicklung der Krankheit und der Geschichte des Kranken in seiner Erkrankung. Geschichtlichkeit und Vergänglichkeit als Perspektiven der Zeitlichkeit können mindestens fragmentarisch in der Geschichte des Kranken als Einheit biografischer Kontinuität und Identität deutlich werden. Der wechselseitige Reduktionszirkel begrenzter Wahrnehmung nur der Krankheit und die Ausblendung der biografischen Perspektive sind nicht wertfrei. Die Beschränkung auf das im Blick auf die Diagnostik und Therapie Gebotene und das dem Kranken Zuträgliche ist aufgehoben in der Achtung der lebensgeschichtlichen Einheit des Kranken. - In dem Therapie-Ziel kann schließlich die Sinnfrage für den Kranken deutlich werden: Zweckorientierte Reparatur von Defekten oder Wiedererlangung und Erhaltung von Gesundheit und akzeptabler Lebensqualität. Darin werden Grundrechte und -ansprüche wie Werte, potentiell auch konflikthaft, deutlich.

Schlüsselwörter: Leiblichkeit - Selbstwahrnehmung - direkte Krankenuntersuchung Krankengeschichte - Therapieziel - Selbstidentität.

Prof. Dr.med. Klaus Gahl Dürerstraße 10

D-38106 Braunschweig 